\title{
Unilateral temporal lobe damage and the partial Kluver-Bucy syndrome
}

\author{
G.D.L. Bates and S.G. Sturman \\ Queen Elizabeth Psychiatric Hospital, Mindelsohn Way, Birmingham B15 2 QZ and City \\ Hospital, Dudley Road, Birmingham B18 7QH, UK
}

\begin{abstract}
The characteristic behavioural constellation of Kluver-Bucy syndrome is believed to be diagnostic of bilateral temporal lobe damage, even in the partial syndrome. The case described is that of a 54 year old woman with gross atrophy of her right temporal lobe following neurosurgery who developed hyperphagia (with driven food-seeking behaviour), placidity and hyposexuality. Her case thereby meets criteria for the partial syndrome but without evidence of bilateral damage. The discussion covers the various presentations of hyperphagia, the significance of the symptom cluster of Kluver-Bucy syndrome and the management difficulties presented by hyperphagia.
\end{abstract}

Keywords: Kluver-Bucy Syndrome - Hyperphagia - Brain Damage - Food-seeking Behaviour - Organic Bulimia

\section{INTRODUCTION}

Since Kluver and Bucy's original description of the behavioural effects of bitemporal lobectomy on rhesus monkeys (Kluver and Bucy 1939), links have been made to human temporal lobe functioning. The full and partial syndromes have been described in humans though never previously with unilateral temporal damage only. The lady described died 12 months after in-patient assessment as a result of myocardial infarction presumed secondary to her non-insulin dependent diabetes and superobesity. The case demonstrates the problems of hyperphagia treatment in addition to the complexities of the neurophysiology of appetite and eating behaviour.

The characteristic behaviour cluster of the KluverBucy syndrome is believed to be pathognomonic of bilateral temporal lobe damage, even in the partial syndrome. The case described is that of 54 year old woman, with gross atrophy of her right temporal lobe following neurosurgery, who developed organic hyperphagia with driven food-seeking behaviour, placidity and hyposexuality, meeting criteria for the partial syndrome with no evidence of bilateral damage. She died 12 months after in-patient assessment as a result of myocardial infarction presumed secondary to her superobesity and non-insulindependent diabetes. The case demonstrates the complexities of the neurophysiology of appetite and eating behaviour and the problems of the treatment of its dysfunction.

\section{CASE REPORT}

A 54 year old right-handed, married Asian woman was admitted for assessment under Section II of the 1983 Mental Health Act. At this time her problems were threefold: insatiable appetite and thirst, apathy and reduced self-care and uncontrolled non-insulindependent diabetes mellitus.

She had been deteriorating at home over an 18 month period but had refused any out-patient appointments for assessment or treatment of her diabetes or behavioural change despite the best efforts of her husband and family doctor. She had agreed to see psychiatrists at home and her compliance with antidepressants, mood stabilizers and oral hypoglycaemics had been confirmed by her husband. Unfortunately, the extent of her hyperphagia in the context of her diabetes had resulted in hyperglycaemia which was life threatening. It was for this reason that application was made for compulsory detention.

Over a 2 year period the amount that she was eating had increased dramatically and she had put on over $60 \mathrm{~kg}$ in weight. She only ate food items though sometimes this would include raw herbs and spices. There was no suggestion of pica.

Over the 18 months prior to admission, the woman had spent the majority of her day in the front room of her house eating sliced bread directly from the bag and drinking milk by the pint. She rarely left the 
room except to find food. She would continue to eat until she vomited and then start eating again. She had no concern for her personal hygiene and her clothes and bed clothes were stained with vomit. The family's attempts to limit her eating reached an extreme when a lock was attached to the kitchen door and a padlock to the door of the refrigerator. Eventually the family found that the most effective strategy was simply not to keep any food in the house although it was extremely inconvenient to do this and she then started to beg for food from their neighbours.

Her second problem, that of apathy and reduced self-care, dated back at least 5 years. Psychiatric assessment at this time recorded that she had become very much more inert and had gradually given up all her household duties, calling on her family to cook, clean and attend to her wants. Her sexual relationship with her husband, which had been previously satisfactory to him, had stopped at around this time. On mental state examination she was described as calm and rather passive. It was recorded that her change in behaviour was in marked contrast to her previous character. She had worked as a headmistress in Pakistan and run a material shop in the UK.

Her third problem, that of diabetes mellitus, was first diagnosed after the onset of her increased eating behaviour. She was seen at home by a consultant physician who prescribed oral hypoglycaemics. She did not alter her dietary habits and unsurprisingly her blood sugar continued to rise despite increased doses. It was considered that her hyperglycaemia was contributing to her inertia and apathy. BM stick measurement prior to admission was in the range of 32-44 $\mathrm{mmol} / 1$ (normal range 4-8 $\mathrm{mmol} / \mathrm{l}$ ).

All these problems had persisted despite compliant treatment with fluoxetine and carbamazepine.

Seventeen years prior to the index admission, a right pterion meningioma had been removed surgically. Histology had shown a largely myxomatous tumour with no evidence of malignancy and she had followed an unremarkable post-operative course. However, 8 years later she had developed an infection of the bone flap. At surgery brain meninges and bone flap were closely adherent and there was necessarily surgical trauma to the right temporal lobe. Post-operatively she suffered two major generalized seizures and persistent vomiting and was discharged on phenytoin and metoclopramide. She remained on phenytoin until time of admission.

She was discharged from neurosurgical follow-up 4 years later at which time her CT scan showed no sign of tumour recurrence or residual sepsis, although expansion of the ventricles on the side of the meningioma was described.
At the time of admission she presented as extremely obese with an asymmetric skull due to her previous surgery. Her clothes were soiled with food debris and vomit. During the course of the interview she ate two apples and a loaf of brown sliced bread. She ate with her hands and crammed the food into her mouth without any social concern.

She appeared frightened but was cooperative with the interview and examination if a firm line was adopted. Her answers to questions were brief and varied between English and Urdu, her native tongue. Her own understanding of her condition was that she felt weak all the time and that eating was the only thing that made her feel strong. She complained that she never felt full. She was aware that she should eat less but did not believe that it was endangering her life and showed no willingness to cut down either her fluid or dietary intake. She made it clear that she was not happy in hospital and that she wanted to leave. However, she made no attempt to do so.

On physical examination she was obese, weighing $120 \mathrm{~kg}$ for her $152 \mathrm{~cm}$. Cognitive testing did not reveal major deficits. She scored 26/30 on the Folstein Mini-mental State which was not suggestive of significant impairment. A neurological opinion was sought. Neurological examination was unremarkable except for a general reduction in limb reflexes. Specifically, there were no visual field defects and she showed no evidence of dominant or non-dominant temporal lobe dysfunction. She had not lost the ability to recognize people. There was no evidence of prosopognosia, apraxia or hemisomatognosia as might be expected from right temporal lobe damage in a right-handed individual. More detailed psychometric testing was not undertaken.

At the time of admission the most urgent concern was control of her hyperglycaemia. Following advice from the local diabetologist, 'sliding scale' insulin therapy was used to bring the blood sugar down to safer levels.

A number of investigations were performed. Full blood count was normal. Urea potassium and creatinine levels were normal but sodium was low at $126 \mathrm{mmol} / 1$ (normal range $134-143 \mathrm{mmol} / 1$ ). Blood glucose was elevated at $31.5 \mathrm{mmol} / 1$ (normal range 5.0-8.7 mmol/1). Urinalysis showed heavy glycosuria but no ketones or proteinuria. Prolactin level was normal at $90 \mathrm{mU} / 1$ (normal range $60-390 \mathrm{mU} / \mathrm{l}$ ) and thyroid stimulating hormone was also normal at $2.23 \mathrm{mU} / 1$ (normal range $0.3-3.8 \mathrm{mU} / 1$ ). Follicular stimulating hormone (FSH) was slightly low at $28.8 \mathrm{IU} / 1$ (normal range $30-118 \mathrm{IU} / 1$ ) but luteinizing hormone levels were normal [27.2 IU/1 (normal range 16-66 IU/1)] as was her urinary cortisol level at 


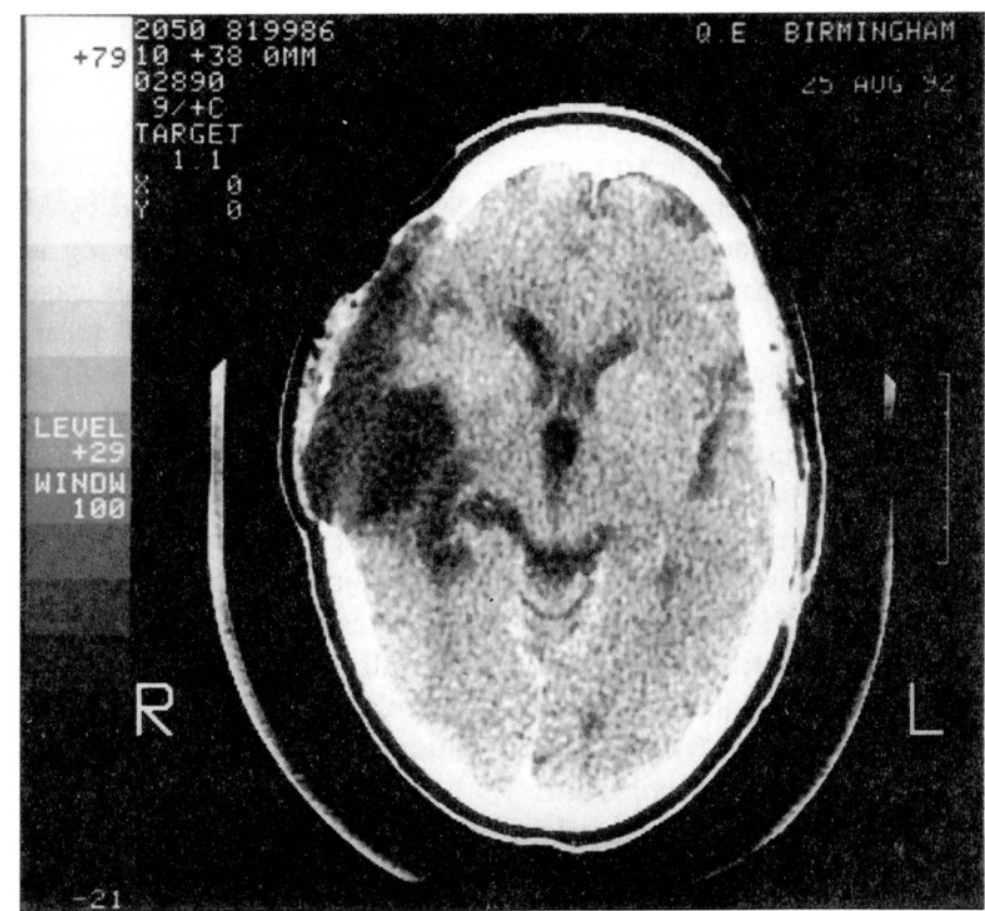

FIG. 1. Brain CT scan.

$96 \mathrm{nmol} / 24 \mathrm{~h}$ (normal range $60-350 \mathrm{nmol} / 24 \mathrm{~h}$ ). Liver function tests and serum albumin were within normal limits. Her low sodium level returned to the normal range once her hyperglycaemia had been corrected. A urine osmolality of $288 \mathrm{mosm} / 1$ indicated that she was able to concentrate her urine when fluid deprived.

A CT scan (Fig. 1) showed absence of the right parietotemporal craniotomy bone flap. Much of the right temporal lobe had been resected or undergone marked atrophy and there was porencephaly affecting the right temporal horn. No pathological enhancement was seen to indicate recurrent meningioma. The pituitary fossa was partially empty but was of normal size. There was no evidence of impaired CSF drainage.

During the first 3 weeks of the patient's admission her behaviour was closely monitored by nursing staff. There was no evidence of depressive symptomatology in terms of sleep disturbance, anhedonia or overall affect. She accepted the restraints put on her eating behaviour, though complained of feeling hungry all the time and would approach various members of hospital staff and patients requesting food. She continued to drink compulsively to such an extent that the handles of the taps of the basin in her room were removed to restrict her unobserved fluid intake.

Initially she spent most of her time on her bed and required great encouragement to wash and attend to her personal hygiene. She did not engage in prolonged conversation with any staff. Despite complaints that she wished to leave, she made no attempts to do so. When her family visited she was overtly critical of their treatment of her and asked them to take her home. Even with family, conversation was limited.

Over 5 weeks, her level of concentration and interaction improved and she became more socially appropriate. She started to play an active role in her diabetic monitoring and started to administer her own insulin. She started to lose weight during the course of her admission and was $6 \mathrm{~kg}$ lighter at time of discharge. Her drinking behaviour persisted despite rectifying her hyperglycaemia.

Our assessment suggested that there was no functional illness or evidence for hypothalamic dysfunction in terms of disordered endocrine function or temperature regulation. Diabetes insipidus was excluded by investigation. Phenytoin toxicity was considered and rejected, although phenytoin has been linked with hyposexuality. It was thought that she suffered from organic hyperphagia and polydypsia secondary to her temporal lobe damage. Clinically, the condition was considered to be irreversible and treatment was based on family support and behaviour containment rather than cure.

These results were discussed with both family and local GP at a series of case conferences. She was 
eventually discharged home with weekday attendance at a mental health resource centre with staff with experience of coping with patients with learning disabilities displaying similarly challenging behaviours.

Six months after discharge improvements in her diabetic control had been maintained and her weight remained steady. She continued to drink water, tea and coffee excessively.

Unfortunately, following a trip back to Pakistan she returned to her original pattern of behaviour. She refused to attend the day hospital and her weight increased. She neglected her diabetic control and stopped injecting her insulin. Within 6 months she was admitted to a general hospital with central chest pain, breathlessness and dizziness. Her ECG on admission showed an acute anterolateral myocardial infarction and she was in left ventricular failure. Her glucose level was $41.4 \mathrm{mmol} / 1$. She was treated with streptokinase and admitted to coronary care unit. The next day she went into electromechanical dissociation and died. A post-mortem request was declined by the family.

\section{DISCUSSION}

Organic hyperphagia is an uncommon condition, rarely seen as an isolated symptom. It occurs in Prader-Willi syndrome, hypothalamic hyperphagia, Kleine-Levin syndrome, Kluver-Bucy syndrome and occasionally in thalamic lesions.

Despite unilateral temporal lobe damage on structural imaging the condition described bears a strong similarity to the Kluver-Bucy syndrome with the prominent apathy, hyperphagia and hyposexuality. It was not possible to image the function of her left temporal lobe, though clinically function was normal.

Kluver-Bucy syndrome was first described in 1939 (Kluver and Bucy, 1939). They reported dramatic behavioural changes in experimental monkeys who had undergone bitemporal lobectomy. Whether the resultant syndrome of hyperphagia, placidity, hyperorality, aberrant sexual behaviour and "psychic blindness" was secondary to removal of limbic structures or temporal neocortex is not yet settled. While there are problems in extrapolating the pathology and clinical features from monkeys to humans, the syndrome has been recognized in a variety of human clinical settings: bitemporal lobectomy, head trauma and encephalitis, particularly of herpes simplex type. The full syndrome is extremely rare with only a few cases described in the literature. However, a variety of partial syndromes are seen.

The first description of Kluver-Bucy syndrome in humans was made by Terzian and Dalle Ore (1955).
A patient developed all symptoms apart from hyperorality following surgical bitemporal lobectomy for control of intractable seizures.

The first complete human case of Kluver-Bucy syndrome was identified by Marlowe et al. (1975) in a 20 year old man following herpes simplex encephalitis. On the basis of his case they suggested several human equivalents of monkey pathology: psychic blindness was equated with visual agnosia; the loss of anger and fear response was expressed as placidity and flattened affect while the increased sexual activity of the monkey was manifested as altered sexual orientation in the human (the patient made appreciative, lewd comments to male nurses); hypermetamorphosis (an irresistible impulse to attend and react to visual stimuli), hyperorality and hyperphagia in humans correlated with increased oral exploratory behaviour and dietary changes in the monkey.

Lilley et al. (1983) reviewed the literature and presented 12 patients with full and partial KluverBucy syndrome. In their series, the breakdown of aetiology was as follows: Pick's disease five, encephalitis four, trauma two, Alzheimer's disease one. They divided human Kluver-Bucy syndrome into six component symptoms: visual agnosia, placidity, altered sexual behaviour, hyperorality, altered dietary habits and hypermetamorphosis. They went on to discuss the significance of the various symptoms and suggested that hypersexuality and sensory agnosias are the least common symptoms of the partial syndrome. The altered sexual behaviour is more usually in the form of inappropriate sexual comments or hyposexuality rather than hypersexuality (Devinsky, 1992). Placidity and dietary/oral changes occurred most commonly. Lilly et al. argued that a minimum of three symptoms comprise the partial syndrome which implies bitemporal dysfunction, having a localizing significance comparable to the entire symptom complex.

A distinction should be made between megaphagia and the complex food-seeking behaviours which may complicate hyperphagia. Most commonly hyperphagia is seen in conditions of hypothalamic pathology. Prader-Willi syndrome results from a translocation involving chromosome 15 and manifests in defects of hypothalamic function causing obesity, shortness, and abnormal temperature control. It is characterized by excessive eating and driven food-seeking behaviour, including begging and stealing both food and money for food. The picture is similar in traumatic hypothalamic injury. Skorwezska et al. (1989) describe the problems of driven food-seeking behaviour in three patients with hypothalamic damage following surgery for craniopharyngioma while Childs (1987) describes three 
such patients in intensive rehabilitation following head injury.

Critchley (1962) described megaphagia as part of the Kleine-Levin syndrome, a condition characterized by periodic episodes of hypersomnolence and increased appetite. Patients consume enormous quantities of food and beverage in a compulsive robotic fashion eating all foods in sight, regardless of quality or quantity.

Patients with Kluver-Bucy syndrome show a range of eating behaviours between megaphagia and driven food-seeking activity, and clearly a continuum exists. However, similar extremes of food seeking to that seen in our patient have been previously described (Lilly et al., 1983) and extremes of food seeking do not appear to have a localizing significance. Hypothalamic pathology was excluded by there being no evidence of disordered temperature or endocrine regulation and normal CT scan appearance.

Given the rarity of organic hyperphagia it is unsurprising that literature relating to treatment is scanty. The case reports that exist suggest that, with the exception of Kleine-Levin syndrome, these syndromes are extremely difficult to treat. Critchley (1962) reports that Kleine-Levin syndrome responds favourably to lithium prophylaxis. The others are universally resistant to behavioural modification, appetite suppressants, lithium and other approaches.

The only promising avenue of research has been into the use of opiate antagonists. Endogenous opiates have been demonstrated to be involved in appetite control (Grandison and Guidotti, 1977). This observation was taken up by Kyriakides et al. (1980), who used naloxone in the treatment of three patients with Prader-Willi syndrome. They found a great reduction in food intake in two of the three patients. Childs (1987) also reported improvement in his three patients with traumatic hypothalamic hyperphagia treated with naltrexone, a long-acting oral opiate antagonist.

\section{CONCLUSION}

This is the first description of a partial Kluver-Bucy syndrome without evidence of bilateral temporal lobe damage. The woman described demonstrates three symptoms usually associated with bilateral temporal lobe damage, namely hyperphagia, placidity and hyposexuality. The partial syndrome of Kluver and Bucy requires a minimum of three symptoms from the following: visual agnosia, placidity, altered sexual behaviour, hyperorality, altered dietary habits and hypermetamorphosis.

The driven food-seeking behaviour is a more com- plex phenomenon which compares with that found in hypothalamic conditions including the Prader-Willi syndrome, but has been previously described in Kluver-Bucy syndrome. It is interesting that her overall inertia was overridden if she was frustrated in obtaining food. Further investigation proved impossible due to the patient's death by a myocardial infarction a year after her admission. This also demonstrates the high mortality rate of this condition, particularly when complicated by diabetes mellitus.

Intervention in the form of correction of her hyperglycaemia and increased social support improved her concentration and sociability. Her polydipsia remained unchanged. In accordance with other reported cases of organic hyperphagia it remained stubbornly resistant to treatment. Drug intervention with naltrexone was not attempted, although in retrospect a therapeutic trial might have been helpful.

\section{Acknowledgements}

The authors thank Dr Martin Davies for his permission to write up this patient under his care, Dr Mike Prendergast for reading the manuscript and Claire Brittain for typing the manuscript.

\section{REFERENCES}

Childs A (1987) Naltrexone in organic bulimia: a preliminary report. Brain Injury, 1, 49-55.

Critchley M (1962) Periodic hypersomnia and megaphagia in adolescent males. Brain, 85, 627-656.

Devinsky O (1992) In: Behavioural Neurology 100 Maxims, pp. 226-228. Edward Arnold, London.

Grandison S and Guidotti A (1977) Stimulation of food intake by muscimol and beta endorphin. Neuropharmacology, 16, 633-636.

Lilly R, Cumming JL, Benson F and Frankel M (1983) The human Kluver-Bucy syndrome. Neurology (Cleveland), 33, 1141-1145.

Kluver H and Bucy PC (1939) Preliminary analysis of functions of the temporal lobes in monkeys. Archives of Neurology and Psychiatry, 42, 979-1000.

Kyriakides M, Silverstone T, Jeffcoate W and Laurance B (1980) Effect of naloxone on hyperphagia in PraderWilli syndrome. Lancet, 816-817.

Marlowe WB, Mancall EL and Thomas TJ (1975) Complete Kluver-Bucy syndrome in man. Cortex, 11, 53-59.

Skorzewska A, Lal S, Waserman J and Guyda H (1989) Abnormal food-seeking behaviour following surgery for craniopharyngioma. Neuropsychobiology, 21, 17-20.

Terzian H and Dalle Ore G (1955) Syndrome of Kluver and Bucy reproduced in man by bilateral removal of temporal lobes. Neurology (Minneapolis), 5, 373-380.

(Received 22 September 1994; accepted as revised 11 May 1995) 


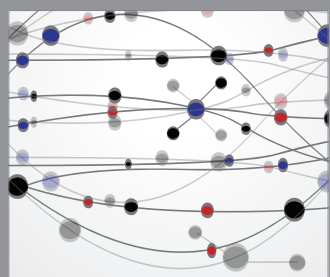

The Scientific World Journal
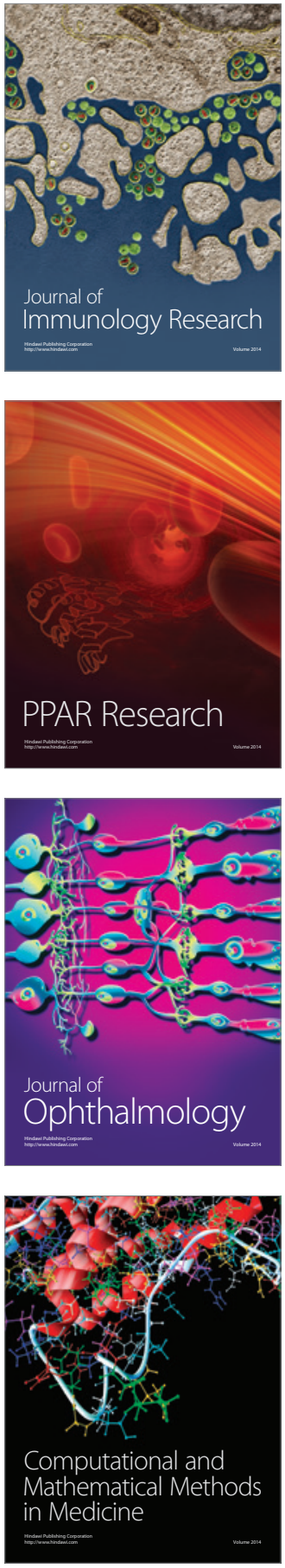

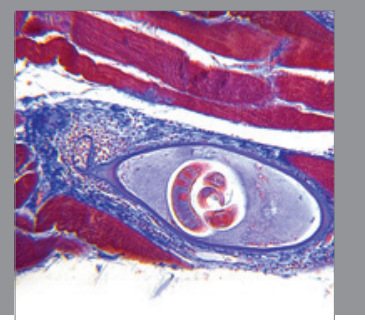

Gastroenterology

Research and Practice
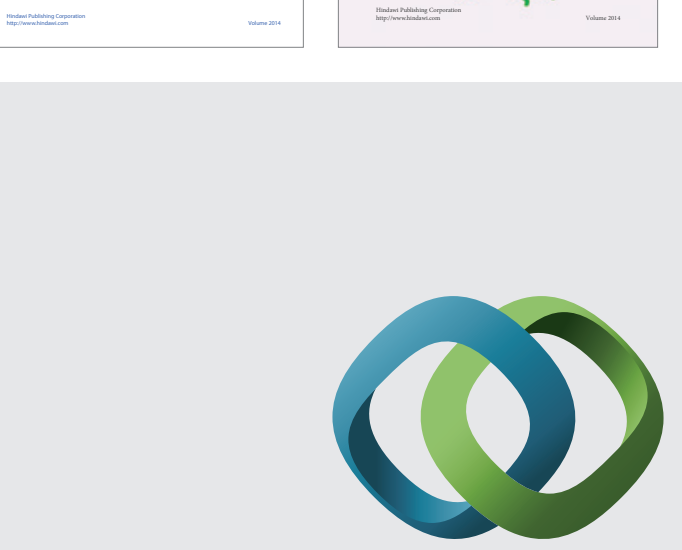

\section{Hindawi}

Submit your manuscripts at

http://www.hindawi.com
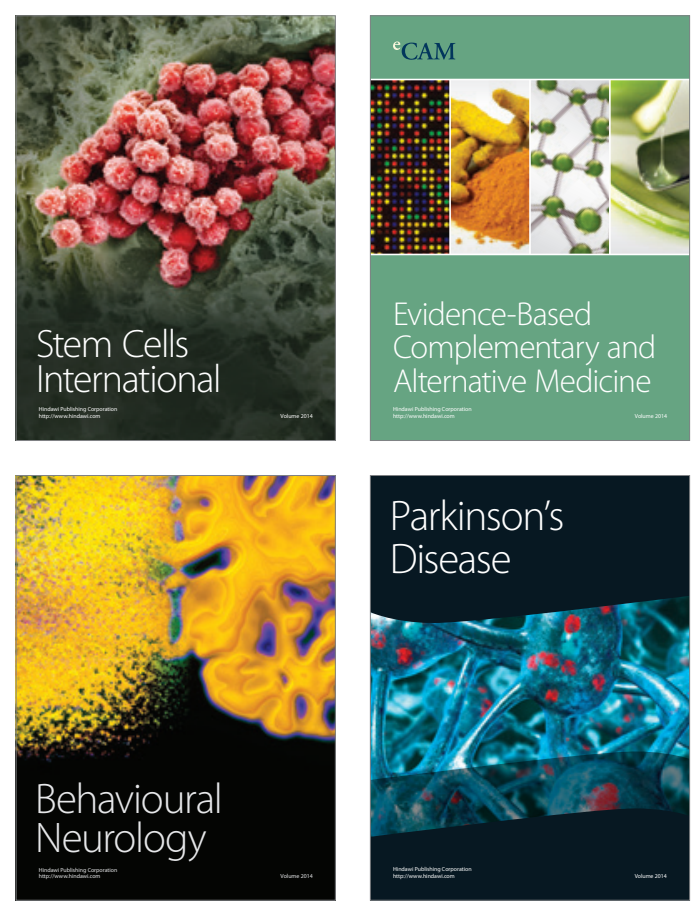

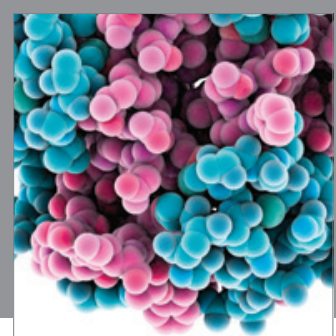

Journal of
Diabetes Research

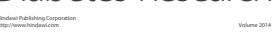

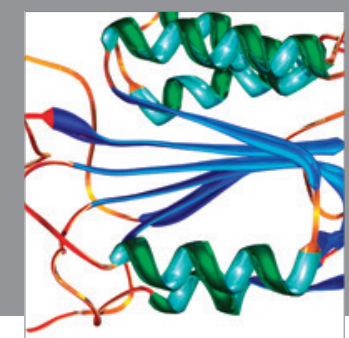

Disease Markers
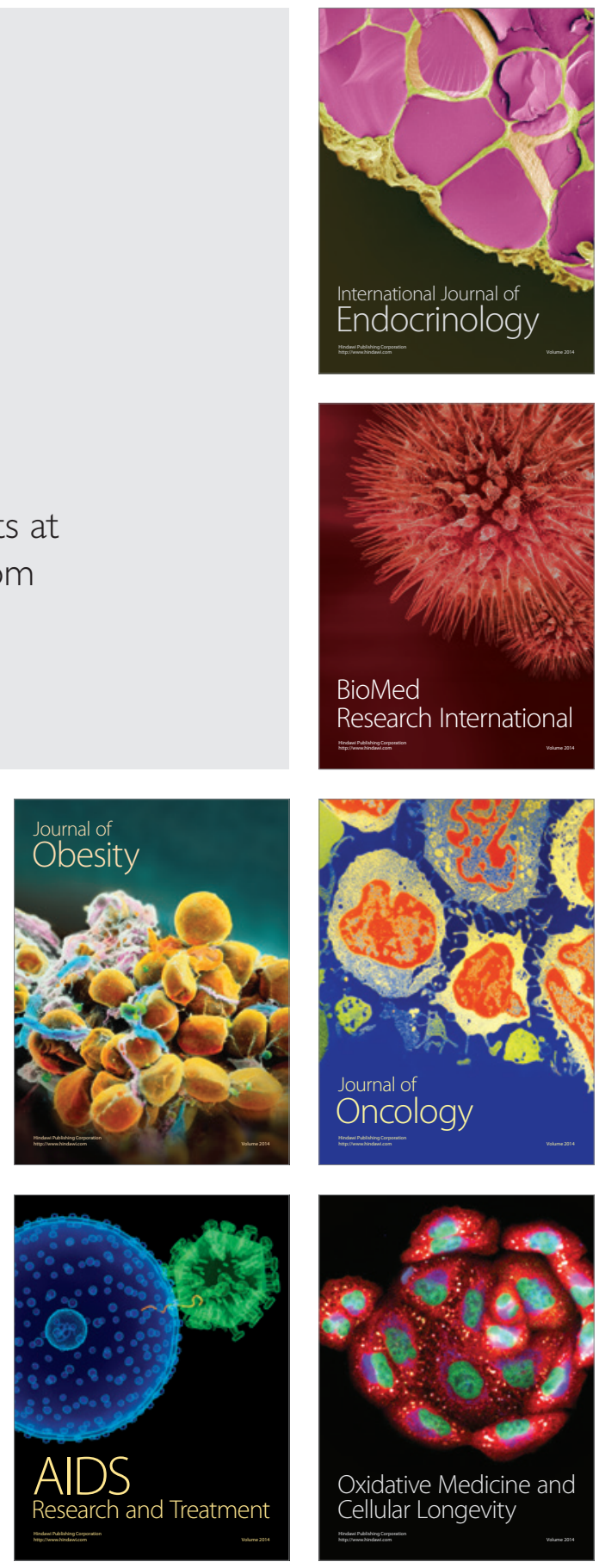